\title{
Analyzing the regulation of metabolic pathways in human breast cancer
}

\author{
Gunnar Schramm ${ }^{1 \dagger}$, Eva-Maria Surmann ${ }^{1 \dagger}$, Stefan Wiesberg ${ }^{2}$, Marcus Oswald ${ }^{2}$, Gerhard Reinelt $^{2}$, Roland Eils $^{1,3^{*}}$, \\ Rainer König ${ }^{1 *}$
}

\begin{abstract}
Background: Tumor therapy mainly attacks the metabolism to interfere the tumor's anabolism and signaling of proliferative second messengers. However, the metabolic demands of different cancers are very heterogeneous and depend on their origin of tissue, age, gender and other clinical parameters. We investigated tumor specific regulation in the metabolism of breast cancer.
\end{abstract}

Methods: For this, we mapped gene expression data from microarrays onto the corresponding enzymes and their metabolic reaction network. We used Haar Wavelet transforms on optimally arranged grid representations of metabolic pathways as a pattern recognition method to detect orchestrated regulation of neighboring enzymes in the network. Significant combined expression patterns were used to select metabolic pathways showing shifted regulation of the aggressive tumors.

Results: Besides up-regulation for energy production and nucleotide anabolism, we found an interesting cellular switch in the interplay of biosynthesis of steroids and bile acids. The biosynthesis of steroids was up-regulated for estrogen synthesis which is needed for proliferative signaling in breast cancer. In turn, the decomposition of steroid precursors was blocked by down-regulation of the bile acid pathway.

Conclusion: We applied an intelligent pattern recognition method for analyzing the regulation of metabolism and elucidated substantial regulation of human breast cancer at the interplay of cholesterol biosynthesis and bile acid metabolism pointing to specific breast cancer treatment.

\section{Background}

Breast cancer is a prevalent disease and a leading cause of cancer death in women [1]. Worldwide, breast cancer is the second most common type of cancer after lung cancer and the fifth most common cause of cancer death. Breast cancer patients with the same stage of disease can have very different treatment responses and overall outcome. Clinical predictive factors like age, tumor size, lymph node status, histological and pathological grade or hormone-receptor status, often fail to accurately predict clinical outcome, distant metastasis and recurrence of the cancer.

\footnotetext{
*Correspondence: r.eils@dkfz.de; r.koenig@dkfz.de

† Contributed equally

'Department of Bioinformatics and Functional Genomics, Institute of Pharmacy and Molecular Biotechnology, and Bioquant, University of Heidelberg, INF 267, 69120 Heidelberg, Germany

Full list of author information is available at the end of the article
}

Chemotherapy and hormonal therapy reduces the risk of distant metastases by approximately one third. However, $70-80 \%$ of the patients would have survived without it (five years of follow-up) [2-4]. A more accurate means of prognosis and selection of therapy would substantially improve disease-free and overall survival of breast cancer patients [5]. Cancer cells acquire their hallmarks of malignancy through the accumulation of advantageous gene activation and inactivation events over long periods of time [6]. Nevertheless, the molecular basis of breast cancer tumorigenesis remains poorly understood. A long-standing strategy for cancer treatment is to attack basic tumor metabolism by inhibiting nucleotide biosynthesis [7,8] and DNA production [9].

Besides this, over the past decade there have been exciting developments in analyzing large scale gene expression profiles. This improved the understanding of the tumors' composition and behavior to develop new targets for therapy [1]. Many studies of gene expression 
identified expression profiles that are prognostic for patients with breast cancer. However, comparisons of the lists of genes derived from these studies showed that they overlap only slightly due to differences in the patient cohorts, microarray platforms, and mathematical methods of analysis [6]. One strategy to tackle this problem is to map lists of differentially expressed genes on groups of genes with related functions according to the information provided by several databases such as Gene Ontology [10] and KEGG [11]. Finding enrichments of specific gene sets related to certain phenotypes or cell states yields a functional grouping of differentially expressed genes which can be related to their pathogenic behavior and can lead to more robust results in comparison to the analysis of single genes.

To detect the enrichment of gene sets, commonly, a list of significantly differentially expressed genes is identified and statistical tests applied, such as Fisher's exact test and $\chi^{2}$ test. In a different approach, a gene-specific statistics, known as the "local" statistics, measures the strength of association between the gene expression and the phenotype for each gene. A global statistics for a gene set is then constructed as a function of local statistics for each gene in it. The significance is assessed by permutation tests [12]. A global test for gene sets to associate gene expression with clinical outcome was presented by Goeman and co-workers and enabled determining whether the global expression pattern of a group of genes is significantly related to clinical outcome of interest using a linear regression approach [13]. In another approach, expression levels of all genes in the gene sets are combined and presented as gene specific features. These features are then compared between the treatment and the control groups to identify significantly affected gene sets [14]. In general, these methods test the association of all genes in a gene set with the phenotypes, whereas often only genes in a subset of the gene set are associated with the phenotype. Some of the genes may not belong to the set due to incompleteness or errors in the available data. Additionally, even if all genes in the gene set have apparently the same function, or belong to the same process, it is likely that only a few genes are associated with the phenotype.

To overcome these and other gene-associated problems, transcriptional data was analyzed using topology information of cellular networks. Topological information derived from the metabolic network was connected by calculating Z-scores of highly correlated sub-networks [15]. Chuang and co-workers improved classification of breast cancers with expression patterns of small subnets of a signal transduction network [16]. Substantial new genetic mediators for prostate cancer were found using reverse engineered gene networks in combination with gene expression profiles [17]. Rapaport and co-workers found gene expression patterns of neighboring genes in the network yielding good classification of the profiled samples by calculating Fourier transformations and rejecting high frequency signals [18]. Common gene expression levels of neighboring nodes in a metabolic network were calculated by averaging over all neighbors of a gene, and revealed several interesting regulated pathways for the human immune system [19]. However, these approaches were not developed to detect highly contrasting expression of neighboring genes that undergo a switch-like shift of regulation in a tumor cell. Importantly, especially these switches can be highly relevant to identify potential drug targets that specifically attack the tumor at nodes at which it redirects fluxes in the network to establish parasitic advantages.

In our study, gene expression profiles of breast tumors having an "unfavorable" prognosis were compared to breast tumors with a "favorable" prognosis. We wanted to track how the aggressive (unfavorable) tumors have specifically regulated their metabolism to optimize their oncogenetic fitness, and to elucidate ways to severely perturb this process. For this, we used an approach that detects orchestrated regulation of neighboring enzymes in the metabolic network. We mapped gene expression data onto optimally arranged grid representations of pathways of the metabolic network and applied Haar wavelet transforms onto defined pathways of the network to combine gene expression values from neighboring enzymes. These combined features were tested using a non-parametric test (Wilcoxon) if they could separate samples from different treatments. Metabolic pathways were selected that had features with the most discriminative gene expression patterns. We detected a substantially higher number of significant gene expression patterns in comparison to commonly used enrichment tests. We revealed 19 significant metabolic pathways including increased purine and pyrimidine biosynthesis which were needed for increased mitosis cycles. Furthermore, we found pathways for increased energy metabolism (glycolysis, pyruvate metabolism and fructose/ mannose metabolism). Interestingly, we observed the regulation of a possible a cellular switch in the pathway for bile acid biosynthesis redirecting the metabolic flux to the synthesis of steroids while preventing degradation into bile acids.

\section{Results and Discussion}

In this study, 250 patients were examined, 196 having a "favorable" and 54 patients an "unfavorable" prognosis. 1826 reactions could be extracted from KEGG [11] for 1771 out of which expression values could be assigned. The workflow of the method is depicted in Figure 1. Pathway maps from KEGG were represented as twodimensional lattice grids with densely packed reactions. 


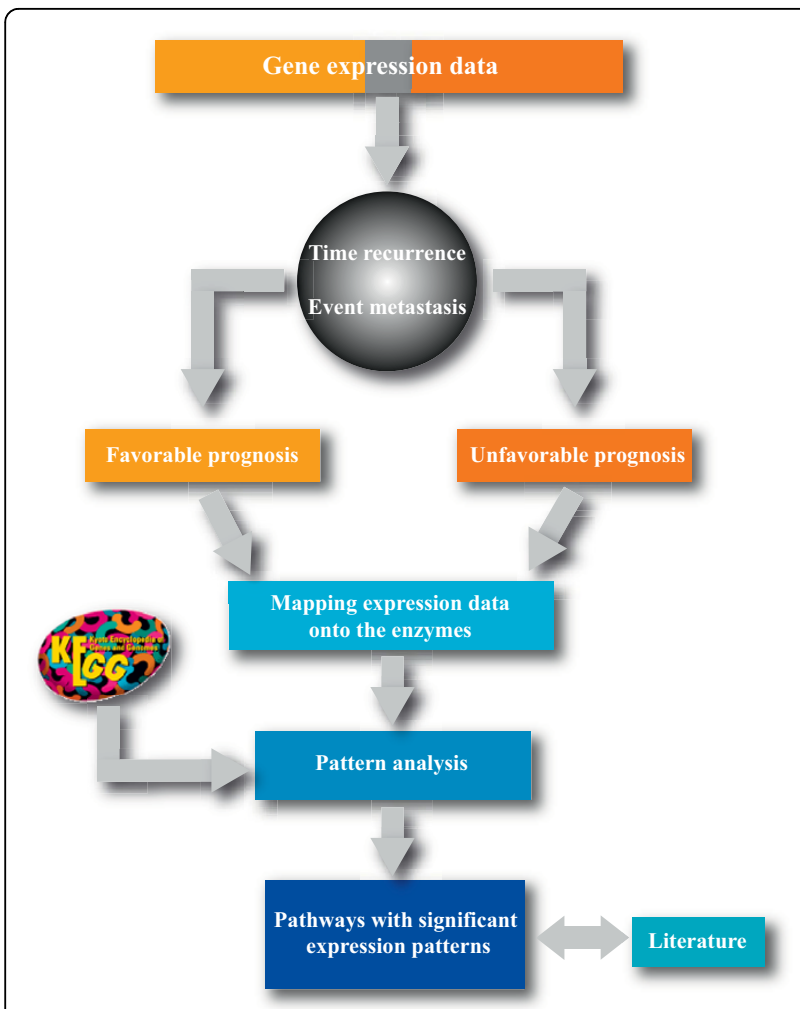

Figure 1 The workflow. Samples were divided into patients with favorable and unfavorable prognosis according to their time of recurrence (time period of relapse after the first event of breast cancer, denoted in years) and event of metastasis (favorable: time recurrence above 5 years, unfavorable: time recurrence of less than three years and the occurrence of metastasis). Expression data were mapped onto the reactions from KEGG and analyzed. Pathways with significant expression patterns were ranked according to the significance of the patterns and compared with the literature.

The reactions were arranged in a way that their neighborhoods in the network were preserved as optimally as possible (using the grid arrangement method, for details see methods). Gene expression data was mapped onto the reactions representing the according expressed enzymes. Wavelet transforms were used to combine expression values of neighboring reactions by all possible combinations of subtractions and additions. The out-coming features were tested (Wilcoxon rank test) for their possibility to discriminate between the two tumor entities (favorable and unfavorable). Figure 2 illustrates the principle of the pattern analysis for an example pathway. Pathways with the best discriminating features were selected. We revealed significant features from 19 different pathways (Table 1), including pyrimidine, purine, aminoacyl-tRNA metabolism, pyruvate metabolism, glycolysis/gluconeogenesis and fructose/ mannose metabolism. These pathways have been expected as they accounted for higher biosynthesis of nucleic acids and proteins and higher energy demands of the aggressive (unfavorable) tumors. We also revealed less expected differentially regulated pathways, such as biosynthesis of steroids and bile acids. We compared the performance of our algorithm with commonly used enrichment methods. Fisher's exact tests revealed only one pathway (Lysine biosynthesis, $\mathrm{P}=0.048$ ) to be significantly enriched with differentially regulated genes ( $P \leq 0.05$, threshold for defining the differentially expressed genes also $P=0.05$ ). In addition, we applied the well established Gene Set Enrichment Analysis (GSEA [20], two sided) to the data yielding also only one significantly enriched pathway, i.e. the pyrimidine metabolism $(\mathrm{P}=2.2 \mathrm{E}-03)$. We compared the performance of our method to Fisher's exact tests and GSEA with simulated data yielding considerably higher sensitivity for our method (see Additional file 1 A2 and Receiver Operator Characteristics: Figure A2). In the following, we will discuss the oncogenetic relevance of the pathways we found.

\section{Pyrimidine and purine metabolism}

Most up-regulated reactions were identified in the pyrimidine $(\mathrm{P}=2.25 \mathrm{E}-04)$ and purine $(\mathrm{P}=1.21 \mathrm{E}-03)$ metabolism. These pathways were up-regulated to enable enforced nucleotide biosynthesis for increased cell cycle activity of the aggressive tumors. Nearly all enzymes involved in the biosynthetic pathway for nucleotides were up-regulated, such as enzymes converting substrates to dNTPs and polyribonucleotide nucleotidyltransferases (EC 2.7.7.7), incorporating dNTPs into DNA. Enzymes reversing pyrimidine and purine anabolism, such as enzymes degrading dNTPs, were downregulated (ECs 3.6.1.17, 1.3.1.2 and 2.7.4.3). Reactions involved in RNA synthesis were partially up-regulated to increase protein biosynthesis (EC 2.7.7.8). Furthermore, reactions which were responsible for synthesizing adenosine were up-regulated (EC 2.4.2.1). Adenosine was shown to be angiogenic, cyto-protective and anti-inflammatory in several tissues and contributed to more aggressive behavior and metastasis of cancer cells [21].

\section{Pathways for energy supply were significantly up- regulated}

We detected significant differential expression patterns in glycolysis $(\mathrm{P}=3.85 \mathrm{E}-03)$, pyruvate $(\mathrm{P}=1.20 \mathrm{E}-02)$ and fructose/mannose $(\mathrm{P}=1.55 \mathrm{E}-02)$ metabolism. They were mostly up-regulated to generate sufficient energy and metabolites for fast-growing cancer cells. Genes of the glycolysis pathway have been found to be over-expressed in a set of 24 cancers, while other pathways showed less consistent up-regulation so far [22]. Glycolysis is increased in cancers and this generates ATP by oxidative phosphorylation $[23,24]$. Furthermore, elevated glycolytic flux may support the production of 


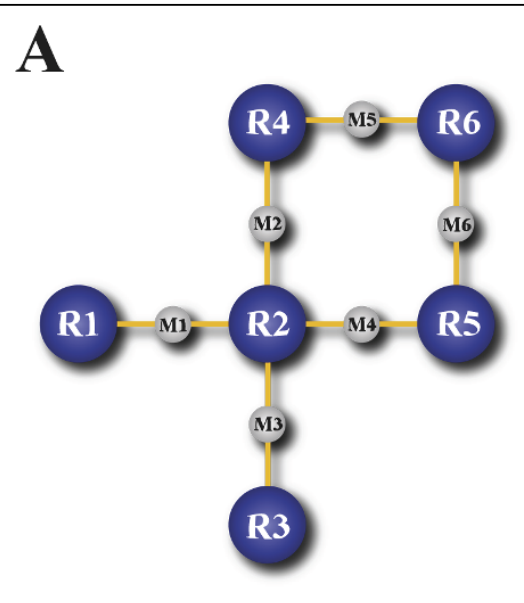

B
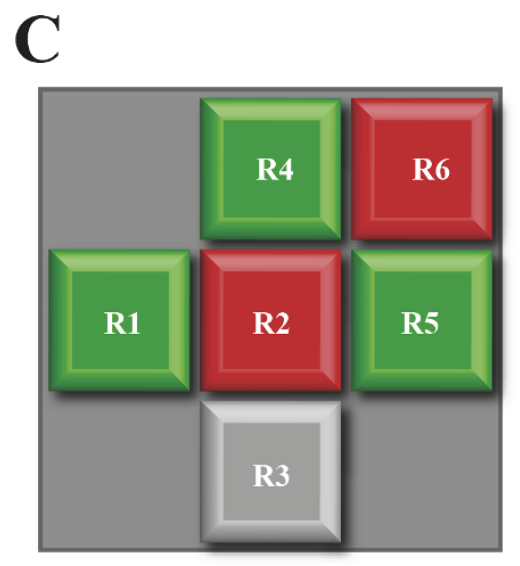

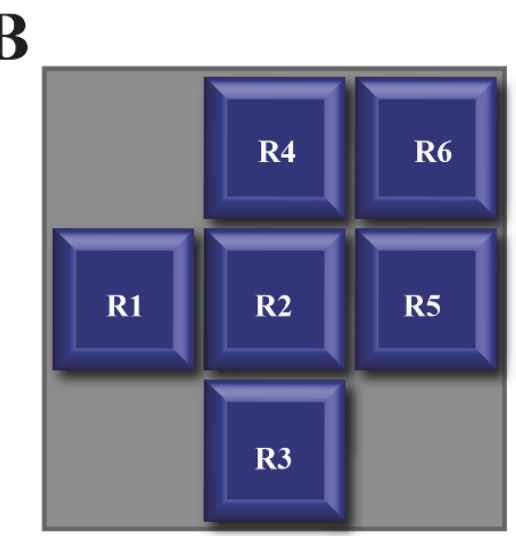

D

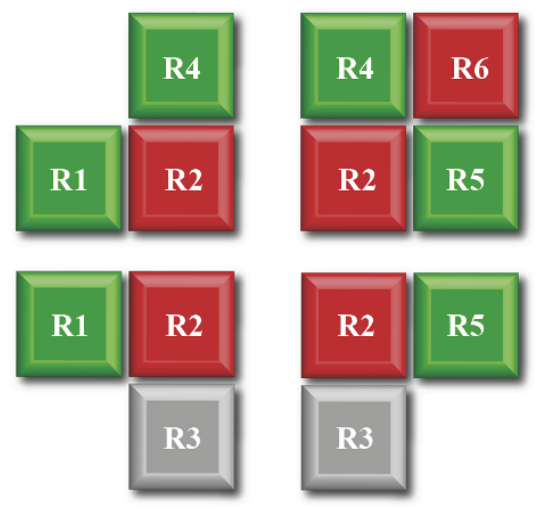

Figure 2 Compiling the features. A. We sketch the method with a simple example pathway consisting of six reactions R1-R6 and six metabolites M1-M6. B. To apply the pattern analysis method (wavelet transforms), the pathway needed to be represented on a two dimensional lattice grid. Reactions were optimally arranged to preserve next nearest neighborhoods while minimizing the distances of neighboring reactions. Metabolites didn't need to be displayed in this representation but rather used to determine the neighborhoods (e.g. R1 is a neighbor of R2 because it produces $\mathrm{M} 1$ which is needed as a substrate for R2 or vice versa). C. Gene expression data was mapped onto the corresponding enzymatic reactions. In this example genes of enzymes for reactions R1, R4, R5 were high expressed and of enzymes for reactions R2 and R6 low expressed. D. Combined gene expression features were assembled by Haar wavelet transforms which basically calculated additive and subtractive combinations of $2 \times 2$ pixels of the grid (pixels without reactions were filled with zeros). The figure shows all four possible arrangements of $2 \times 2$ pixels for which the wavelet transforms were calculated. The same procedure was done for all tumor samples. The feature which best separated the tumor entities (favorable from unfavorable) was selected for the significance of this pathway.

important metabolites that contribute to essential cellular processes like fatty acid synthesis, nucleotide synthesis and to the better protection of oxidative stress $[25,26]$.

However, the extent of up-regulated genes in glycolysis varies for different cancer types. In our study, all except one differentially regulated reactions were upregulated, including enzymes such as glyceraldehyde-3phosphate dehydrogenase (EC 1.2.1.12), phosphor glycerate mutase (EC 5.4.2.1), phosphoglycerate kinase (EC 2.7.2.3), triosephosphate isomerase (EC 5.3.1.1) and fructose-bisphosphate aldolase (EC 4.1.2.13). These enzymes were also described as up-regulated by Altenberg and co-workers [22]. Inhibitors of glycolysis, such as the glucose analog 2-deoxyglucose, which binds and suppresses hexokinase I, and arsenate, that causes arsenolysis in glyceraldehyde-3-phosphate dehydrogenase, as well as 3-bromopyruvate, an inhibitor of hexokinase II, have already been developed to target this metabolic abnormality and could effectively kill cancer cells [27].

Inhibition of glycolysis was effective even in killing cancer cells with a multidrug resistance (MDR) phenotype. It is known that cells expressing MDR proteins require ATP as their energy source to export drugs out of the cell. Thus, to overcome this drug resistance, depletion of cellular ATP causes the excretion to fail and consequently, cancer cells become more sensitive to anti-cancer therapy [27]. 
Table 1 Significantly differentially regulated pathways

\begin{tabular}{|c|c|c|c|c|c|c|}
\hline Rank & Pathway & $\begin{array}{r}\text { Differentially } \\
\text { regulated }\end{array}$ & $\begin{array}{l}\text { Number of } \\
\text { reactions }\end{array}$ & $\begin{array}{c}\text { Down-regulated } \\
\text { in unfavorable }\end{array}$ & Up-regulated in unfavorable & P-value \\
\hline 1 & Histidine metabolism & 6 & 14 & 3 & 3 & $1.15 \mathrm{E}-05$ \\
\hline 2 & Alanine and aspartate metabolism & 8 & 19 & 2 & 6 & $1.56 \mathrm{E}-05$ \\
\hline 3 & Valine, leucine and isoleucine degradation & 13 & 36 & 10 & 3 & 3.74E-05 \\
\hline 4 & Pyrimidine metabolism & 37 & 75 & 3 & 34 & 2.25E-04 \\
\hline 5 & Fatty acid metabolism & 14 & 36 & 9 & 5 & 2.48E-04 \\
\hline 6 & Biosynthesis of Steroids & 10 & 41 & 2 & 8 & $3.12 \mathrm{E}-04$ \\
\hline 7 & Methionine metaboplism & 4 & 12 & 2 & 2 & 7.36E-04 \\
\hline 8 & Purine metabolism & 17 & 91 & 3 & 14 & $1.21 \mathrm{E}-03$ \\
\hline 9 & Glycine, Serine and Threonine metabolism & 12 & 31 & 4 & 8 & $1.28 \mathrm{E}-03$ \\
\hline 10 & Propanoate metabolism & 4 & 18 & 3 & 1 & $2.12 \mathrm{E}-03$ \\
\hline 11 & Lysine Biosynthesis & 6 & 6 & 5 & 1 & 2.33E-03 \\
\hline 12 & Aminoacyl-tRNA biosynthesis & 5 & 20 & 1 & 4 & 2.83E-03 \\
\hline 13 & Glycolysis/Gluconeogenesis & 17 & 32 & 1 & 16 & $3.85 \mathrm{E}-03$ \\
\hline 14 & Bile acid biosynthesis & 10 & 28 & 4 & 6 & $5.26 \mathrm{E}-03$ \\
\hline 15 & Pentose phosphate pathway & 5 & 20 & 1 & 4 & $8.84 \mathrm{E}-03$ \\
\hline 16 & Inositol phosphate metabolism & 8 & 25 & 1 & 7 & $9.75 \mathrm{E}-03$ \\
\hline 17 & Pyruvate metabolism & 6 & 27 & 2 & 4 & $1.20 \mathrm{E}-02$ \\
\hline 18 & Fructose and mannose metabolism & 11 & 23 & 1 & 10 & 1.55E-02 \\
\hline 19 & Galactose metabolism & 8 & 24 & 4 & 4 & $2.00 \mathrm{E}-02$ \\
\hline
\end{tabular}

Pathways with significant regulation patterns and more than three differentially regulated reactions (as defined by KEGG), bold: pathways which had also significant patterns in the second analyzed dataset.

We found a smaller pattern of up-regulated reactions in the pyruvate metabolism. Significantly up-regulated were: pyruvate kinase (EC 2.7.1.40) which is responsible for ATP production in glycolysis, and the conversion of pyruvate to malate via (S)-Malate:NADP+ oxidoreductase (ECs 1.1.1.38, 1.1.1.39, 1.1.1.40). Aldehyde dehydrogenase (EC 1.2.1.3) was down-regulated to prevent degradation of fatty acids. Oxaloacetate was reported to directly induce cell proliferation by increasing DNA synthesis [28]. Surprisingly, our results did not show any increase in lactate dehydrogenase, which is often upregulated in cancer cells [22]. Highly expressed reactions in fructose and mannose metabolism were mainly involved in the conversion of D-fructose (EC 4.1.2.13), D-fructose-1P (ECs 2.7.1.1, 2.7.1.11, 4.1.2.13) and D-mannose (ECs 2.7.1.1, 2.7.1.11, 4.1.2.13) into glyceraldehyd-3P, a substrate of glycolysis, leading to increased ATP production.

\section{Biosynthesis of steroids and bile acids}

The steroid pathway was mainly up-regulated in breast cancer of unfavorable outcome. 8 out of 10 reactions were significantly up-regulated (8 reactions from KEGG comprising ECs 2.5.1.29, 2.5.1.10, 2.5.1.1, 1.1.1.170, 5.3.3.5, 1.3.1.21). Cholesterol is a precursor for the biosynthesis of steroid hormones. It was shown that sex steroid hormones such as estrogen increase the proliferation of breast cancer cells by acting on estrogen receptors [29]. Directly connected with the production of cholesterol is the biosynthesis of bile acids. Figure 3 shows the regulation of bile acid biosynthesis (including the results from the second analyzed dataset, the second analyzed dataset is described in the next section). In the bile acid biosynthesis pathway, reactions generating cholesterol and its derivates were up-regulated (ECs 3.1.1.13 and 2.3.1.26), whereas the lower part of the pathway was mainly down-regulated, i.e. the production of bile acids such as cholate and lithocholate (ECs 2.3.1.16, 1.2.1.3). Conversion of cholesterol to bile acids is the major pathway for cholesterol catabolism in the human body [30]. Therefore, down-regulation of the lower part of the bile acid pathway and up-regulation of cholesterol biosynthesis may, in conjunction, support steroid biosynthesis which supports estrogen mediated tumorigenesis of breast cancer cells.

Besides this, there have been intensive cancer investigations on bile acids as they have very heterogeneous effects on tumor cells and carcinogenesis. They are known enhancers for invasiveness of colon cancers [31,32]. In turn, bile acids can induce apoptosis either specifically (receptor-mediated interactions) [33] or, in high concentrations, non-specifically (as detergents) through mitochondrial destabilization and oxidative stress [34]. Non-toxic doses of deoxycholic acid (DCA), chenodeoxycholic acid (CDCA) and lithocholic acid (LCA) induced differentiation in promyelocytic leukemia cell lines [35]. 6ECDCA is a synthetic bile acid derivative and can act as a selective FXR ligand to promote 


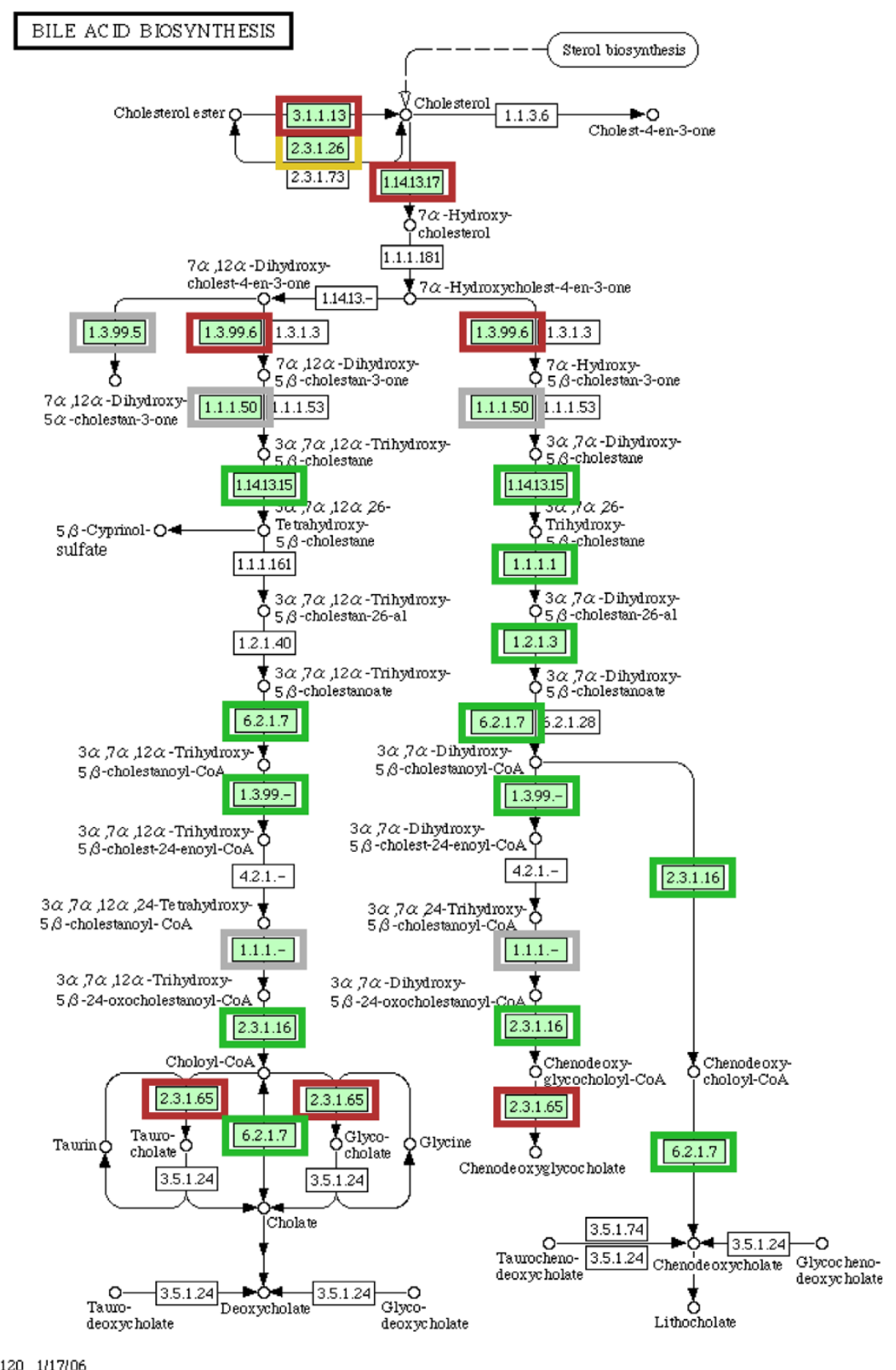

Figure 3 Regulation of the pathway for bile acid biosynthesis of both analyzed datasets. Red frame indicates up-regulation in at least one dataset, green frame down-regulation in at least one dataset, yellow frame up-regulation in the two respective datasets, and grey frame downregulation in the two datasets, respectively, and grey frame no differential regulation in both datasets. The map was taken from KEGG [11]. The lower part of this pathway was mainly down-regulated to prevent degradation of steroids into bile acids, whereas the upper part was mainly up-regulated to support steroid metabolism.

differentiation of preadipocyte cell lines [35]. In the blood plasma of postmenopausal women with newly diagnosed breast cancer, elevated concentrations of DCA were detected which may have been released from osteoblasts to induce migration of the cancer [30]. This would explain a down-regulation in breast cancer cells as they may use elevated bile acid levels from the blood for detaching and infiltrating while sustaining their endogenous cholesterol synthesis.

Bile acids are normally predominantly produced by hepatocytes which may explain the elevated levels of bile acids in the blood when elevated breast cancer 
estrogen is decomposed into bile acids in the liver. In conclusion, this regulation may reflect a cellular switch which redirects the metabolic flux from degradation of cholesterol (via bile acids) into the conversion of steroid hormones, suggesting drug targets in the biosynthesis of steroids, such as mevalonate (diphospho) decarboxylase (EC 4.1.1.33) and mevalonate kinase (EC 2.7.1.36).

\section{Analyzing a second dataset}

To verify our findings we analyzed an independent gene expression dataset of breast cancer [36,37] consisting of 414 samples. The data was downloaded from Array Express (GSE6532, http://www.ebi.ac.uk/microarray-as/ ae/) and normalized as described in Methods. We divided the dataset into 250 tumors showing a favorable prognosis and 61 tumors with an unfavorable prognosis following our criteria as described for the other dataset (in Methods). 103 tumors were discarded as they did not fit in any of the prognostic groups. The significance level was again set to $\mathrm{P}=0.05$. Our analysis revealed 12 pathways with significantly differentially regulated patterns (see Additional file 1 Table A1). Although the overlap of survival predicting genes between different breast cancer studies is often rather small [38], four pathways from the first analyzed study also showed a significant regulation pattern in this second dataset, i.e. histidine metabolism, valine, leucine and isoleucine degradation, bile acid biosynthesis and galactose metabolism. Interestingly also the citrate cycle showed a significant regulation pattern which compares to the pathways for carbon hydrate metabolism we found in the first analyzed dataset, and $\mathrm{C}_{21}$-steroid hormone metabolism which compares to steroid biosynthesis found in the first analyzed study.

\section{Conclusion}

Performing a network based gene expression analysis revealed interesting insights into the metabolism and regulation of aggressive breast tumor cells. Expected differentially regulated pathways in cancer could be confirmed, e.g. the aggressive tumors showed significantly up-regulated pathways for purine and pyrimidine synthesis to maintain elevated proliferation, as well as the upregulation of glycolysis and pyruvate metabolism to support energy supply for the tumor. The analysis revealed insights into differentially regulated metabolic pathways in breast cancer cells, which may support the induction of proliferation by inositol signal transduction cascades (inositol phosphate metabolism) and steroid hormones (biosynthesis of steroids). An interesting view in breast cancer metabolism was observed in the interplay of biosynthesis of steroids and bile acids, the latter of which was down-regulated possibly to convert cholesterol into proliferative acting steroid hormones. Such a cellular switch would be interesting to compare to other tumors and tissues, also in order to define a tumor specific therapy. Performing Fisher's exact tests as a standard enrichment analysis revealed the lysine biosynthesis pathway to be significantly enriched with differentially expressed genes. The GSEA method yielded pyrimidine biosynthesis. In addition to this pathway, our method yielded eighteen further significant regulation patterns. However, not all pathways detected by such enrichment tests and also our pattern analysis may be relevant for the pathogenesis of the analyzed disease. Some pathways in our results, such as valine, leucine and isoleucine degradation, could not be associated with oncogenesis and may need further examination.

Complex regulated pathways which were relevant to breast tumors with unfavorable prognosis were detected and described in a straightforward manner. The global analysis of network patterns offered a good insight into the regulation of metabolism in breast tumors and may support revealing new potential targets for drug design, specifically in the interplay of the biosynthesis of bile acids and steroids.

\section{Methods}

We wanted to identify pathways and regions of pathways that showed patterns of differentially regulated reactions. To find such patterns we applied wavelet transforms on the mapped gene expression data. For the application of wavelet transforms and the subsequent feature calculations we needed to arrange the metabolic pathways onto a 2-dimensional grid (Figure 2 illustrates this procedure with a small example pathway).

\section{Preparing the microarray data}

Normalized gene expression data was taken from a published study [5] of breast-cancer samples of 295 women (diagnosis between 1984 and 1995) with age $\leq 53$ years and no previous history of cancer, except for non-melanoma skin cancer. The data was downloaded from Rosetta Inpharmatics at http://www.rii.com/publications/2002/ nejm.html. The gene expression profiles were derived by using oligonucleotide microarrays from Agilent Technologies http://www.agilent.de. Data on relapse-free survival (defined as the time to the first event) and overall survival were available for all patients. Most of the patients had breast cancer of stages one and two. 165 had received local therapy alone, 20 had received tamoxifen only, 20 had received tamoxifen plus chemotherapy, and 90 had received chemotherapy only. 151 patients were diagnosed as lymph-node-negative and 144 as lymph-node-positive. The tumors were primary invasive breast carcinoma that were less than $5 \mathrm{~cm}$ in diameter at pathological examination [5]. To differentiate between tumors with "favorable" and "unfavorable" prognosis, samples were separated 
according to their "time recurrence" (period of time of relapse after the first event of breast cancer, denoted in years) and "event of metastasis" (if metastasis occurs during this period). A time recurrence above 5 years indicated a "favorable" prognosis (196 patients), whereas samples were classified as samples with unfavorable prognosis if they showed a time recurrence of less than three years and the occurrence of metastasis (54 patients). Samples with ambiguous information were discarded ( 45 samples) and identified either by a time recurrence of less than three years without the event of metastasis or by a time recurrence above three years but below five years. A Remark: A new ethical approval was not needed for our study as all our analyses based on published microarray datasets (which also applies to the second analyzed dataset).

\section{Assembling the metabolic pathways}

Pathways were defined according to curated pathway maps of the KEGG database [11] (version from February $\left.4^{\text {th }}, 2009\right)$. Two reactions were neighbors if a metabolite existed that was the product of one and the substrate of the other. We defined reactions as the nodes and metabolites as the edges between them. Pathways without any connected reaction were discarded. This resulted in 99 pathways with 1826 different reactions. Each pathway was represented by its adjacency-matrix. An entry at row $a$ and column $b$ was set to one if there existed a metabolite that was produced by reaction $a$ and consumed by reaction $b$ or vice versa. The sizes of the symmetric adjacency-matrices were between $2 \times 2$ and $92 \times$ 92 reactions.

\section{Ordering the two-dimensional pathway representation with the grid arrangement method}

To apply our feature extraction method we required a 2-dimensional grid arrangement of the metabolic network. We calculated an embedding of the metabolic networks for every KEGG pathway into a 2-dimensional, regular square lattice grid. To preserve neighborhood characteristics of the reactions, we were looking for embeddings in which adjacent nodes of the network were placed onto the grid as close to each other as possible. We wanted to determine an optimal neighborhood in which the total edge length of the graph was minimized according to some metric on the lattice. The network topology was preserved as good as possible. For this purpose the minimization of total edge length is more suitable than a minimization of the longest edge which is widely applied in very large scale integration (VLSI) designs as the latter one allows a variety of optimal solutions in which adjacent nodes are placed unnecessarily far from each other. As a measure of distance in the lattice, we used the natural metric induced by the underlying lattice graph, the so-called Manhattan distance. That is, for any two grid points $u=\left(i_{1}, j_{1}\right)$ and $v=\left(i_{2}, j_{2}\right)$ the distance was given by $d_{u v}=\left|i_{1}-i_{2}\right|+\mid j_{1}$ $-j_{2} \mid$. This resulted in an NP-hard combinatorial optimization problem. We stated this problem as an integral linear program (IP) (see [39] for an introduction to integer programming). We formulated the IP by introducing 3 -dimensional binary variables $x_{v i j}$ for every node $v$ and every grid point $(i, j)$ stating whether or not node $v$ has to be placed on grid point $(i, j)$. For each pair of nodes $(u, v)$ we calculated their distance $d_{u v}$. For a given lattice grid $g$, the undirected network graph $G=(V, E)$ with node set $V$, edge set $E$ and adjacency matrix $M$, the most basic IP was given by finding an optimum for

$$
\min _{x, d} \sum_{a, b \in V, a<b} M(a, b) \cdot d_{a b}
$$

with the constraints

$$
\begin{aligned}
& \sum_{(i, j) \in g} x_{v i j}=1 \quad \text { for all } v \in V, \\
& \sum_{v \in V} x_{v i j} \leq 1 \quad \text { for all }(i, j) \in g \\
& d_{a b} \geq A+B, \quad d_{a b} \geq A-B \\
& d_{a b} \geq-A+B, \quad d_{a b} \geq-A-B
\end{aligned}
$$

for all $(a, b) \in V \times V, a<b$, where

$$
\begin{aligned}
& A:=\sum_{(i, j) \in g} i \cdot x_{a i j}-\sum_{(i, j) \in g} i \cdot x_{b i j \prime} \\
& B:=\sum_{(i, j) \in g} j \cdot x_{a i j}-\sum_{(i, j) \in g} j \cdot x_{b i j}, \\
& x_{v i j} \geq 0, \quad x_{v i j} \in Z \text { for all } v \in V,(i, j) \in g .
\end{aligned}
$$

Constraints Eq. (2) and Eq. (3) guaranteed that all nodes (reactions of the pathway) were placed exactly once and that each grid point could be used at most once, thus avoiding multiple placements and stacking of reactions on a single grid point. Constraints Eq. (4) and Eq. (5) ensured that the distance of node $a$ and $b$ is given by $|A|+|B|$ where $A$ and $B$ are computed by Eq. (6) and Eq. (7) as $A=i_{a}-i_{b}$ and $B=j_{a}-j_{b}$. All variables were enforced to values 0 or 1 by constraint Eq. (8). The problem was solved by CPLEX 8.1 (ILOG, Gentilly, France) for 99 lattice grids (representing 99 KEGGmaps) with an average optimality of $96 \%$ for embeddings 
on square grids of side length $\sqrt{|V|}+1$, rounded up to the next integer. By choosing a grid of the smallest possible size, we reduced both the number of variables in the model and the number of unoccupied sites on the grid. This basic model was enhanced by a number of graph dependent, additional constraints on the distance variables. They provided lower bounds for the distance sums of well-known sub-graph motifs. For an edge induced sub-graph $G^{\prime} \subset G$ with a least objective function contribution of $l b\left(G^{\prime}\right)$, the following inequality was added or dynamically separated by

$$
\sum_{(u, v) \in E\left(G^{\prime}\right)} d_{u v} \geq l b\left(G^{\prime}\right)
$$

The right-hand sides $l b\left(G^{\prime}\right)$ for the different sub-graph motifs needed to be determined only once as they are independent of G. Furthermore, the motifs were defined in a pre-processing step and could therefore be separated quickly during the optimization process. We considered the sub-graph motifs of star graphs, cliques consisting of up to 10 vertices and odd cycles $(2 k+1$ cycles) for $\mathrm{k}=1,2$. Moreover, a certain class of trees with maximum vertex degree $\Delta(\mathrm{T}) \leq 4$ decreased computation time and enhanced separation ability. Furthermore, calculation time was reduced by symmetry breaking constraints eliminating all but a few representative embeddings from each equivalence class of symmetrical embeddings. For this, grid symmetries due to translation, rotation and reflection of the embeddings were considered as well as vertex subsets which inner permutations didn't change the value of the objective function. After solving the optimization problem the values of the variables $x_{v i j}$ allowed to construct the embedding of the metabolic pathway on a 2-dimensional grid placing reaction $v$ on the grid point with coordinates $(i, j)$. On the grid representations of the pathways we then applied our feature generation method.

\section{Pattern recognition of gene regulation on the metabolic network}

Neighboring enzymes on the 2-dimensional grid representation were grouped by combining their gene expression values with wavelet transforms. These transforms yielded combined expression values ("features") of low pass filters to detect similar expression changes and high pass filters to detect contrasting regulation patterns. The wavelet calculation is described in the following. Each cluster-matrix was divided into $2 \times 2$ pixeled disjoint sub-sections (e.g. a cluster matrix of size $8 \times 8$ was divided into 16 sub-sections). Clusters with non-fitting sizes (e.g. $3 \times 3,5 \times 5$,) were extended with rows and columns of zeros to yield matrices that could be divided into $2 \times 2$ pixels sub-sections. For each sub-section, all combinations of row-wise and columnwise mean and differences, respectively, were calculated. This yielded 4 combined values for each $2 \times 2$ pixels sub-section: 1st: mean of the mean of the upper and mean of the lower row, 2nd: difference of the mean of the upper and the mean of the lower row, 3rd: mean of the difference of the upper and the difference of the lower row, and, 4th: difference of the difference of the upper and the difference of the lower row. All four combined values for each $2 \times 2$ pixels sub-section were stored and applied as features for the classifier. This was done for all sub-sections of the matrix. All 1st combined values (mean of means) were taken for a new matrix and were again grouped into $2 \times 2$ fractions that were combined in the same manner, yielding again 4 new features for every fraction. This procedure was repeated until no further grouping was possible. The discriminative behavior of all non-trivial features was tested using Wilcoxon rank tests. Pathways were ranked according to their best discriminating features. Features were regarded as significant if they had $\mathrm{p}$-values $\leq 0.05$ after correction for multiple testing using the Bonferroni method $[40,41]$. Only pathways with more than three significantly, differentially regulated reactions and genes were further investigated to focus on the most relevant features. Two reactions that consisted of exactly the same genes were counted as one reaction.

\section{Additional material}

Additional file 1: Additional results and performance assessment Results for the additional gene expression data set and comparison of our approach with standard enrichment tests using simulated data.

\section{Acknowledgements}

This work was funded within the BMBF-FORSYS consortium Viroquant (\#0313923), the Helmholtz Alliance on Systems Biology and the Nationales Genom-Forschungs-Netz (NGFN+) for the neuroblastoma project, ENGINE.

\section{Author details}

'Department of Bioinformatics and Functional Genomics, Institute of Pharmacy and Molecular Biotechnology, and Bioquant, University of Heidelberg, INF 267, 69120 Heidelberg, Germany. ${ }^{2}$ Interdisciplinary Center for Scientific Computing, University of Heidelberg, 69120 Heidelberg, Germany. ${ }^{3}$ Theoretical Bioinformatics, German Cancer Research Center, INF 580, 69121 Heidelberg, Germany.

\section{Authors' contributions}

GS and RK conceptualized and designed the method. GS and EMS analyzed the data. SW, MO and GR provided solutions for the combinatorial optimization problem. GS, EMS and RK wrote the manuscript. RE and RK revised it critically. All authors read and approved the final manuscript.

\section{Competing interests}

The authors declare that they have no competing interests.

Received: 2 December 2009 Accepted: 10 September 2010 Published: 10 September 2010 


\section{References}

1. Oakman C, Bessi S, Zafarana E, Galardi F, Biganzoli L, Di Leo A: New diagnostics and biological predictors of outcome in early breast cancer Breast Cancer Research 2009, 205:1-11.

2. van 't Veer $L$, Dai $H$, van de Vijver MJ, He YD, Hart AA, Mao M, Peterse $H L$, van der Kooy K, Marton MJ, Witteveen AT, Schreiber GJ, Kerkhoven RM, Roberts C, Linsley PS, Bernards R, Friend SH: Gene expression profiling predicts clinical outcome of breast cancer. Nature 2002, 415:530-536.

3. Polychemotherapy for early breast cancer: an overview of the randomised trials. Early Breast Cancer Trialists' Collaborative Group. Lancet 1998, 352:930-942.

4. Tamoxifen for early breast cancer: an overview of the randomised trials. Early Breast Cancer Trialists' Collaborative Group. Lancet 1998, 351:1451-1467.

5. Van de Vijver MJ, He YD, van't Veer LJ, Dai H, Hart AAM, Voskuil DW, Schreiber GJ, Peterse JL, Roberts C, Marton MJ, Parrish M, Atsma D, Witteveen A, Glas A, Delahaye L, van der Velde T, Bartelink H, Rodenhuis S, Rutgers ET, Friend SH, Bernards R: A Gene-Expression Signature as a Predictor of Survival in Breast Cancer. The New England Journal of Medicine 2002, 347:1999-2009.

6. Fan C, Oh DS, Wessels L, Weigelt B, Nuyten DSA, Nobel AB, van't Veer LJ, Perou CM: Concordance among Gene-Expression-Based Predictors for Breast Cancer. The New England Journal of Medicine 2006, 355:560-569.

7. Chen $L$, Pankiewicz KW: Recent development of IMP dehydrogenase inhibitors for the treatment of cancer. Curr Opim Drug Discov Devel 2007, 10:403-412.

8. Lui MS: Biochemical pharmacology of acivicin in rat hepatoma cells. Biochem Pharamcol 1982, 31:3469-3473.

9. Pedersen-Bjergaard J: Risk of acute nonlymphocytic leukemia and preleukemia in patients treated with cyclophosphamide for nonHodgkin's lymphomas. Comparison with results obtained in patients treated for Hodgkin's disease and ovarian carcinoma with other alkylating agents. Ann Intern Med 1985, 103:195-200.

10. Harris MA, Clark J, Ireland A, Lomax J, Ashburner M, Foulger R, Eilbeck K, Lewis S, Marshall B, Mungall C, Richter J, Rubin GM, Blake JA, Bult C, Dolan M, Drabkin H, Eppig JT, Hill DP, Ni L, Ringwald M, Balakrishnan R, Cherry JM, Christie KR, Costanzo MC, Dwight SS, Engel S, Fisk DG, Hirschman JE, Hong EL, Nash RS, Sethuraman A, Theesfeld CL, Botstein D, Dolinski K, Feierbach B, Berardini T, Mundodi S, Rhee SY, Apweiler R, Barrell D, Camon E, Dimmer E, Lee V, Chisholm R, Gaudet P, Kibbe W, Kishore R, Schwarz EM, Sternberg P, Gwinn M, Hannick L, Wortman J, Berriman M, Wood V, de la Cruz N, Tonellato P, Jaiswal P, Seigfried T, White R: The Gene Ontology (GO) database and informatics resource. Nucleic Acids Res 2004, 32:D258-261.

11. Kanehisa M, Araki M, Goto S, Hattori M, Hirakawa M, Itoh M, Katayama T, Kawashima S, Okuda S, Tokimatsu T, Yamanishi Y: KEGG for linking genomes to life and the environment. Nucleic Acids Res 2008, 36: D480-484.

12. Pitman EJG: Significance tests which may be applied to samples from any population. Royal Statistical Society Supplement 1937, 4:119-130, 225232.

13. Goeman JJ, van de Geer SA, de Kort F, van Houwelingen HC: A global test for groups of genes: testing association with a clinical outcome. Bioinformatics 2004, 20:93-99.

14. Yan $X$, Sun F: Testing gene set enrichments for subset of genes: SubGSE. BMC Bioinformatics 2008, 9:1-15.

15. Patil KR, Nielsen J: Uncovering transcriptional regulation of metabolism by using metabolic network topology. Proc Natl Acad Sci USA 2005, 102:2685-2689.

16. Chuang HY, Lee E, Liu YT, Lee D, Ideker T: Network-based classification of breast cancer metastasis. Mol Syst Biol 2007, 3:140.

17. Ergun A, Lawrence CA, Kohanski MA, Brennan TA, Collins JJ: A network biology approach to prostate cancer. Mol Syst Biol 2007, 3:82.

18. Rapaport F, Zinovyev A, Dutreix M, Barillot E, Vert JP: Classification of microarray data using gene networks. BMC Bioinformatics 2007, 8:35.

19. Nacu S, Critchley-Thorne R, Lee P, Holmes S: Gene Expression Network Analysis, and Applications to Immunology. Bioinformatics 2007.

20. Mootha VK, Lindgren CM, Eriksson KF, Subramanian A, Sihag S, Lehar J, Puigserver P, Carlsson E, Ridderstrale M, Laurila E, Houstis N, Daly MJ, Patterson N, Mesirov JP, Golub TR, Tamayo P, Spiegelman B, Lander ES, Hirschhorn JN, Altshuler D, Groop LC: PGC-1alpha-responsive genes involved in oxidative phosphorylation are coordinately downregulated in human diabetes. Nat Genet 2003, 34:267-273.

21. Spychala J: Regulation and function of ecto-5'-nucleotidase and adenosine in cancer. 39 th Meeting of the Polish Biochemical society 2003, 185

22. Altenberg B, Greulich $\mathrm{KO}$ : Genes of glycolysis are ubiquitously overexpressed in 24 cancer classes. Genomics 2004, 84:1014-1020.

23. Guppy M, Greiner E, Brand K: The role of the Crabtree effect and an endogenous fuel in the energy metabolism of resting and proliferating thymocytes. Eur J Biochem 1993, 212:95-99.

24. Pfeiffer T, Schuster S, Bonhoeffer S: Cooperation and competition in the evolution of ATP-producing pathways. Science 2001, 292:504-507.

25. Feron O: Pyruvate into lactate and back: from the Warburg effect to symbiotic energy fuel exchange in cancer cells. Radiother Oncol 2009, 92:329-333.

26. Vander Heiden MG, Cantley LC, Thompson CB: Understanding the Warburg effect: the metabolic requirements of cell proliferation. Science 2009, 324:1029-1033.

27. Xu R, Pelicano H, Zhou Y, Carew JS, Feng L, Bhalla KN, Keating MJ, Huang P. Inhibition of glycolysis in cancer cells: a novel strategy to overcome drug resistance associated with mitochondrial respiratory defect and hypoxia. Cancer Research 2005, 65:613-621.

28. Li Y: Oxaloacetate induces DNA synthesis and Mitosis in primary cultured Rat Hepatocytes in the basence of EGF. Biochemical and Biophysical Research communications 1993, 193:1339-1346.

29. Suzuki T, Miki Y, Nakamura Y, Moriya T, Ito K, Ohuchi N, Sasano H: Sex steroid-producing enzymes in human breast cancer. Endocrine-Related Cancer 2005, 12:701-720.

30. Zimber A, Gespach C: Bile acids and derivatives, their nuclear receptors FXR, PXR and ligands: role in health and disease and their therapeutic potential. Anticancer Agents Med Chem 2008, 8:540-563.

31. Debruyne PR, Bruyneel EA, Li X, Zimber A, Gespach C, Mareel MM: The role of bile acids in carcinogenesis. Mutat Res 2001, 480-481:359-369.

32. Pai R, Tarnawski AS, Tran T: Deoxycholic acid activates beta-catenin signaling pathway and increases colon cell cancer growth and invasiveness. Mol Biol Cell 2004, 15:2156-2163.

33. Garewal H, Bernstein H, Bernstein C, Sampliner R, Payne C: Reduced bile acid-induced apoptosis in "normal" colorectal mucosa: a potential biological marker for cancer risk. Cancer Res 1996, 56:1480-1483.

34. Katona BW, Anant S, Covey DF, Stenson WF: Characterization of Enantiomeric Bile Acid-induced Apoptosis in Colon Cancer Cell Lines. The journal of biological chemistry 2009, 284:3354-3364

35. Zimber A, Chedeville A, Gespach C, Abita JP: Inhibition of proliferation and induction of monocytic differentiation on HL60 human promyelocytic leukemia cells treated with bile acids in vitro. Int $J$ Cancer 1994, 59:71-77.

36. Loi S, Haibe-Kains B, Desmedt C, Lallemand F, Tutt AM, Gillet C, Ellis P, Harris A, Bergh J, Foekens JA, Klijn JG, Larsimont D, Buyse M, Bontempi G, Delorenzi M, Piccart MJ, Sotiriou C: Definition of clinically distinct molecular subtypes in estrogen receptor-positive breast carcinomas through genomic grade. J Clin Oncol 2007, 25:1239-1246.

37. Loi S, Haibe-Kains B, Desmedt C, Wirapati P, Lallemand F, Tutt AM, Gillet C, Ellis P, Ryder K, Reid JF, Daidone MG, Pierotti MA, Berns EM, Jansen MP Foekens JA, Delorenzi M, Bontempi G, Piccart MJ, Sotiriou C: Predicting prognosis using molecular profiling in estrogen receptor-positive breast cancer treated with tamoxifen. BMC Genomics 2008, 9:239.

38. Ein-Dor L, Kela I, Getz G, Givol D, Domany E: Outcome signature genes in breast cancer: is there a unique set? Bioinformatics 2005, 21:171-178.

39. Nemhauser G, Wolsey L: Integer and Combinatorial Optimization John Wiley \& Sons, Hoboken 1999

40. Bonferroni CE: II calcolo delle assicurazioni su gruppi di test. Studi in Onore del Professore Salvatore Ortu Carboni. Rome, Italy 1935, 13-60.

41. Gordi T, Khamis H: Simple solution to a common statistical problem: interpreting multiple tests. Clin Ther 2004, 26:780-786.

\section{Pre-publication history}

The pre-publication history for this paper can be accessed here: http://www.biomedcentral.com/1755-8794/3/39/prepub

doi:10.1186/1755-8794-3-39

Cite this article as: Schramm et al:: Analyzing the regulation of metabolic pathways in human breast cancer. BMC Medical Genomics 2010 3:39. 